Th. Palberg

R. Simon

M. Würth

P. Leiderer

\section{Colloidal suspensions as model liquids and solids}

this in the structure-dependent selfdiffusion dynamics. Due to the mesoscopic interparticle spacing the suspensions are easily probed by Bragg-scattering of visible light. This is used to determine the crystallization kinetics and the elastic properties of the resulting colloidal solids. The rigorous control of experimental parameters allows for quantitative tests of theoretical concepts. In the case of elasticity and diffusion, we detect strong systematic influences of the sample morphology. We also discuss how the determined equilibrium properties may contribute to the understanding of recent experiments in the presence of strong shearing fields.

Key words Ordered colloidal suspensions - self-diffusion solidification $=$ elasticity phase diagram

\author{
T. Palberg $(\bowtie) \cdot$ R. Simon \\ M. Würth P. Leiderer \\ Universität Konstanz \\ Fakultät für Physik \\ 78434 Konstanz, FRG
}

Received: 1 February 1994

\section{Introduction}

Colloidal science deals with dispersions of particles in a second medium showing typical length scales of $10^{-8}$ to $10^{-5} \mathrm{~m}$. This may refer to either or both the particle diameter and spacing. Obviously, this definition includes molten or solubilized polymers and macromolecules as well as micellar aggregates, foams, membranes, emulsions and also rigid particles of arbitrary shape. Situated at the intersection of chemistry, biology and physics colloidal science therefore draws from each to form a (by no means homogeneous) interdisciplinary forum, which has attracted continously growing attention over the last decades [1]:

One particular system, suspensions of latex spheres in aqueous electrolytes, may be synthesized with welldefined mean diameters and surface properties. Dilute samples with very small variations in particle properties, so-called monodisperse colloids, have been widely used as model systems within the extremely heterogeneous class of colloidal suspensions which are of prime technical and biological importance [2-4]. The introduction of additional complications, like hydrodynamic interaction which becomes increasingly impor- 
tant at higher volume fractions, or like charge and size polydispersity leading to significant changes in the observed structure factors, facilitates a steady approach to industrially relevant suspensions.

On the other hand, such suspensions show fluid or crystalline ordered phases under certain experimental conditions of strong interparticle interaction. For charged systems these are observed for high surface charge number $Z-10^{3}$, a low concentration of clectrolyte $c \leqslant 10^{-5}$ mol $l^{-1}$ and a sufficiently high volume fraction $\Phi \geqslant 0.005$ [4-6]. Since typical particle spacings then are on the order of the wavelength of visible light, most structural and dynamical properties of these systems are accessible by optical techniques. Although this socalled soft condensed matter exhibits a number of specific and interesting differences, like the low particle density of only $10^{12} \mathrm{~cm}^{-3}$ or diffusional relaxation times in the millisecond range, it behaves strikingly similar to atomic liquids or solids in many respects [1-4].

In recent publications a number of effects have been reported and measuled quantitatively, which are not always easily investigated in atomic substances, either because of inaccessible time scales or because experimental procedilles are vily expensive in time and costs. Examples include nucleation and crystal growth processes from an undercooled melt $[5,6]$. non-equilibrium phase transitions [7-10], transverse [1 1] and longitudinal [12] latlice vibrations, structural relaxation processes [13], and two-dimensional phenomena [14]. These experiments are supported by extensive theoretical work [15-20].

In this paper, we will give a short outline of some of the interesting features observed in strongly correlated colloidal suspensions and their integration into a quantitative description using a Yukawa potential and comparably simple concepts from condensed matter physics. In particular, we will illustrate the self-diffusion dynamics observed under equilibrium conditions in different regions of the phase diagram with special emphasis on the isotropic fluid phase. We will then turn to the equilibrium astic properties of the bec phase, which, in addition to their systematic dependence on the experimental parameters, are highly sensitive to the sample morphology Illustrating the step towards nonequilibrium conditions, we show some recent results on resonant stick-slip phenomena observed in the presence of an external flow freld. We finally report a study on the kinctics of the first-order phase transition from the metastable (shear-)melt to the solid state to demonstrate the principal way of access to a quantitative modeling of general shear-induces: phenomena.

\section{Phase diagram of charged suspensions}

The phase behavior of charge-stabilized colloidal suspensions has recieved much interest in the past few years
[21]. Unlike atomic systems, the temperature is kept constant and the ratio of thermal encrgy to the cnergy of interaction is adjusted by varying experimental parameters: i) the concentration of electrolyte $c_{\mathrm{s}}$ added to the saltfree suspension; ii) the particle number density $\rho$, sometimes also expressed as volume fraction $\Phi=$ $\rho(4 / 3) \pi a^{3}$, where $a$ is the radius of the particles; and. to a minor extent, iii) the particle charge $Z$ by suitable chemical treatment $[23,24]$. We restrict ourselves here to i) and ii) only. noting that recently also the phase diagram in dependence on the particle charge has been recorded [24].

In the case of dilute suspensions $(\Phi \leqslant 0.01)$ of highly charged particles $(Z \geqslant 300$ at $a \approx 100 \mathrm{~nm}$ ) hydrodynamic and van der Waals interaction may safely be ignored. Thus, both the strength and the range of the interaction are determined by a Yukawa pair potential $V(r)$ :

$V(r)=U \frac{e^{-x r}}{r}$

where $r$ is the interparticle distance, $U$ the surface potential and $x$ the Debye screening paramtcr. A convenient expression for $U$ and $x$ is given by the so-called modified DLVO approximation (MDA) [25] which has recently been verified expcrimentally by a dynamical test of interaction potentials [26]:

$U_{\mathrm{MDA}}=\frac{Z_{\mathrm{PBC}}^{2} e^{2}}{4 \pi \varepsilon \varepsilon_{0}}\left(\frac{\exp \left(\varkappa_{\mathrm{MDA}} a\right)}{\left(1+\varkappa_{\mathrm{MDA}} a\right)}\right)^{2}$,

and

$\gamma_{\mathrm{MDA}}^{2}=e^{2}\left(2 p_{4}+\rho Z_{\mathrm{PBC}}\right) / k_{\mathrm{B}} T$.

(Here $e$ denotes the elementary charge, $\varepsilon \varepsilon_{0}$ the dielectric permittivity of the suspending medium, $k_{\mathrm{B}}$ the Boltzmann constant, and $\rho_{\mathrm{s}}=2000 N_{\mathrm{A}} c_{\mathrm{b}}$ the concentration of excess electrolytc with $N_{\mathrm{A}}$ being the Avogadro number.) $Z_{\mathrm{PBC}}$ is a renormalized surface charge [15] used both in the calculation of the MDA pair potential and the MDA screening parameter, which is calculated via the numerical solution of the non-linearized PoissonBoltzmann equation for a particle in a spherical WignerSeitz cell and the subsequent fit of a Yukawa potential. Obviously, the systematic variation of the interaction calls for a prccise control of the experimental parameters. The standard procedure to prepare ordered suspensions so far has been the introduction of a small amount of ion exchange resin directly into the measuring cell. Very often, interesting nonequilibrium phenomena, like the separation into layered regions of different structure, are observed during deionization $[10,27]$. After a few weeks the suspension is rendered practically free of salt ions. 
Systematic experiments in dependence on $\Phi$ are then performed by adding further particles; to alter the salt concentration by the addition of electrolyte the suspension has to be transferred to another cuette free of ion exchange resin. These procedures are time consuming and somewhat tedious for extreme caution has to be taken to exclude contamination with $\mathrm{CO}_{2}$.

Recently, a conceptually different fast and accurate deionization procedure has been reported by us, which is also used in the control of experimental parameters throughout this paper [28]. The suspension is peristaltically driven through a closed teflon tubing system connecting a separate ion exchange chamber, a reservoir under inert gas atmosphere to add salt or particles, two measuring chambers for in situ control of particle concentration (static light scattering or transmission) and salt concentration (conductivity) and one or more actual measuring cells. The deionization times are reduced by over two orders of magnitude, its completion is monitored, and simultaneous measurements are easily possible on the same suspension.

The accuracy of the procedure is demonstrated in Fig. 1 [29] where we show the phase diagram of the suspension of latex spheres used for all of the experiments presented here. These commercially available particles (Seradyn, IN, USA; Lot 2011M9R) have a hydrodynamic diameter of $a_{\mathrm{h}}=102 \mathrm{~nm}$, a titrated number of surface groups $N_{\mathrm{SO}_{4}}=950$ of which only $Z=580$ are dissociated at $\Phi=0.003$ due to the overlap of the electric double layers [30]. The resulting renormalized charge (which is to be inserted into Eq. (2) a, b) is $Z_{\mathrm{PBC}}=395$.

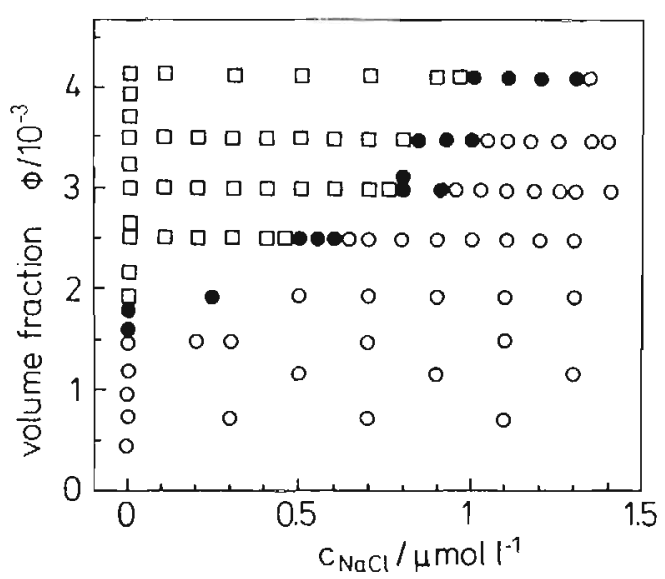

Fig. 1 Phase diagram of $102 \mathrm{~nm}$ polystyrene spheres carrying $N$ $=950$ sulfate surface groups in dependence on the $\mu$ molar concentration $c_{\mathrm{s}}$ of aqueous electrolyte and the volume fraction $\Phi$ [29]. Note the low values of $c_{5}$ and $\Phi$ which are precisely controlled by the new preparation technique and considerably extend the previously studied parameter space for these particles [31]. The symbols are: squares: bcc-crystal; open circles: fluid; filled circles: coexistence bec-fluid
We note that the accuracy reached is on the order of $1-2 \%$ at volume fractions $\Phi \approx 0.0001$ and $\mu$ molar salt concentrations.

The experiment extends the known phase diagram of these particles [31] to extremely low concentrations of particles and salt. As can be seen, the equilibrium phase diagram shows a fluid and a bcc crystalline region separated by a coexistence regime widening with increasing $\Phi$ and $c_{\mathrm{s}}$. The freezing and melting line obtained by a polynomial fit agree well with recent theoretical calculations $[32,16]$.

If the experimental parameters are fixed at a point in the bcc region, several differently ordered modifications may be prepared. Firstly, the suspension is left in a metastable shear molten state of fluid order directly after stopping its flow through the tubing system. From this it readily crystallizes to form a wall-nucleated, quasiepitaxially grown, oriented monolithic crystal surrounding a core of unoriented polycrystalline material [29]. Gentle shear processing allows the preparation of samples with only one of these morphologies. It further facilitates the development of preferred orientation (shear-texturing) or control of the grain size, depending on the details of the shear-processing. In fact, a quasi nanocrystalline situation may be reached, where each single crystallite having a diameter on the order of $30 \mu \mathrm{m}$ contains only as few as $10^{5}$ particles (at $\rho=3 \cdot 10^{18} \mathrm{~m}^{-3}$ ).

\section{Structurally determined self diffusion}

The transport properties of colloidal suspensions have long been of interest. Changes in the fundamental processes of diffusional transport due to the formation of cages of neighboring particles are observable in model suspensions with high sensitivity by light-scattering techniques $[1-4,11,19,20,23]$. We herc employ the Forced Rayleigh Scattering (FRS) technique to determine the self- or tracer-diffusion coefficients $D_{\mathrm{L}}$ for the longtime limit and its dependence on the strength of interaction, the structure and on morphological details [33, 34, 26].

A small fraction of the particles are UV-sensitized with 6-Nitro-BIPS (Merck, FRG). An absorption grid of variable wavelength is formed in the sample by two crossed UV-laser beams. Its diffusional decay is monitored via the Bragg reflection of a HeNe laser beam. The monoexponential signal is evaluated for the self diffusion coefficient $D_{\mathrm{L}}$, using independently measured corrections for the incoherent and coherent scattering backgrounds and the relaxation time of the dye. Up to 20 single measurements are averaged in a run to obtain an error in the value of $D_{\mathrm{L}}$ of less than $1 \%$.

In the presence of fluid order the time-dependent mean squared displacement $\left\langle r^{2}\right\rangle$ of a colloidal sphere in a 
medium of viscosity $\eta$ shows two linear regimes. At short times the particle diffuses freely and the self-diffusion coefficient in this regime $D_{\mathrm{s}}=\left\langle r^{2}\right\rangle / 6 t$ takes the StokesEinstein value $D_{0}=k_{\mathrm{B}} T / 6 \pi \eta \mathrm{a}$ [35]. Any direct repulsive interaction with other particles considerably reduces the diffusional transport for longer times. The structural relaxation of the cage of neighboring particles surrounding the particle becomes the limiting process of particle diffusion. After several rearrangements of the cage a second linear regime of $\left\langle r^{2}\right\rangle$ is reached, where the long time self-diffusion coefficient $D_{\mathrm{L}}$ is found to decrease with increasing strength of interaction.

It is convenient to express the decrease of $D_{\mathrm{L}}$ in terms of the self-diffusion ratio $D_{\mathrm{L}} / D_{\mathrm{S}}$, which is 1 for a noninteracting system. In Fig. 2, we plot this ratio versus the volume fraction for differently ordered phases [33]. The upper curve shows the data continuing smoothly from the equilibrium fluid phase to the shear molten state. This characterizes the latter phase as an undercooled melt by a dynamic measurement. We will first concentrate on the behavior observed in the fluid ordered states and come back to this figure in the discussion of the solid phases.

Recent Brownian Dynamics simulations on Yukawa fluids have shown that the diffusion ratio has a universal value of $D_{\mathrm{L}} / D_{\mathrm{S}}=0.098$ at the freezing transition of colloidal fluids [34]. Since the Yukawa potential as expressed in Eq. (1) approaches the limits of a hard sphere system for large screening and of the one-component plasma for negligible screening, this constitutes a dynamical freezing criterion for Brownian systems of arbitrary Yukawa parameters, which is similar in spirit to the well-known Lindemann rule or the Hansen-Verlet criterion.

In Fig. 3, we show the results of FRS measurements for several experimental runs over a wide range of parameters in comparison with the phase boundaries (solid lines) of our system. We present only the coordinates of samples with $D_{\mathrm{L}} / D_{\mathrm{S}}=0.09 \pm 0.010$ and further discriminate between those showing deviations on the order of the experimental error $\left(D_{\mathrm{L}} / D_{\mathrm{S}}=0.098 \pm\right.$ $0.05)$ and those of higher or lower values. The coexistence region coincides with the region of $D_{\mathrm{L}} / D_{\mathrm{S}}=$ $0.098 \pm 0.05$. The diffusion ratio is remarkably constant along the freezing line. This universality is surprising, since dynamic quantities usually depend much more sensitively on the details of the interaction than their static counterparts. In fact the value of $D_{\mathrm{L}} / D_{\mathrm{s}}$ changes rapidly across the phase transition. This sensitivity was recently also used to gain a more detailed insight into the particle interaction [26]. We have compared data sets on the entire fluid phase measured under equally well defined experimental conditions to the results of Brownian dynamics simulations in order to discriminate between alternative Yukawa potentials. In particular, the self-diffusion ratio can be quantitatively predicted without any

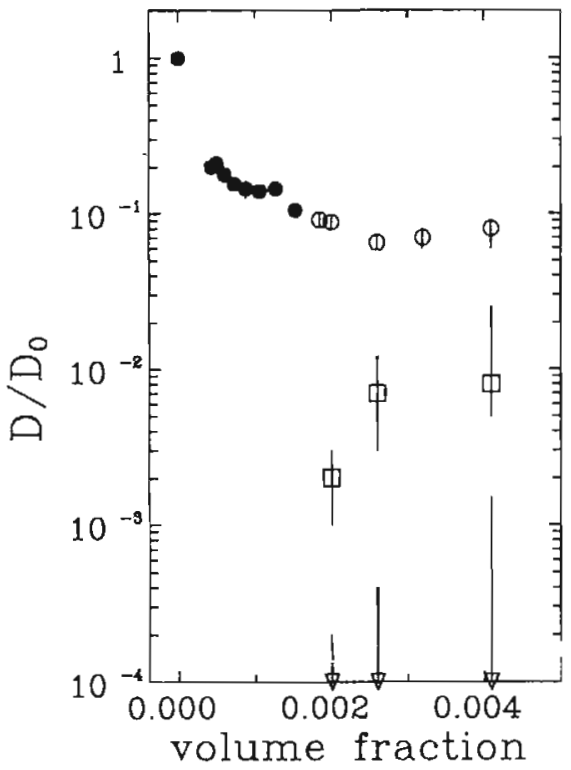

Fig. 2 Sele-diffusion ratio $D_{\mathrm{L}} / D_{\mathrm{S}}$ as measured by FRS in completely deionized samples in dependence on the volume fraction $\Phi$. The symbols denote the differently ordered phases: filled circles: equilibrium fluid; open circles: undercooled melt; squares: untextured polycrystalline bcc solid; triangles: upper bound for $D_{\mathrm{L}} / D_{\mathrm{S}}$ in oriented monolithic bec crystals. $D_{\mathrm{L}} / D_{\mathrm{S}}$ decreases with increasing $\Phi$ in the liquid phases but increases in the polycrystalline phase [33]

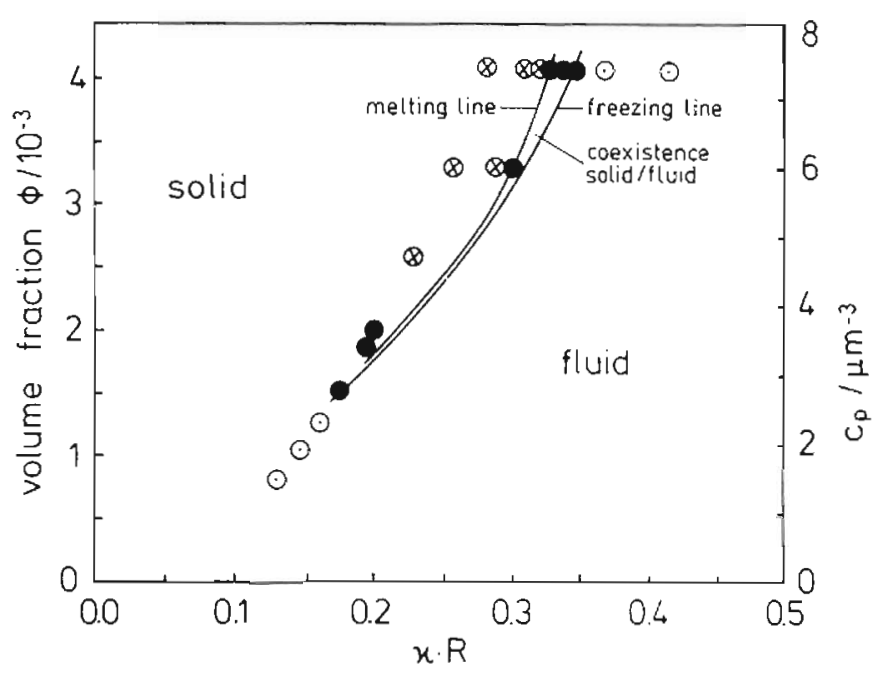

Fig. 3 Phase diagram of the same particles in terms of their volume fraction and the dimensionless screening paramter $* a$. The solid curves represent the freezing and melting lines derived from Fig. 1. Symbols show the coordinates of samples with $D_{\mathrm{L}} / D_{\mathrm{S}}$ fulfilling the following criteria: $00.093 \leqslant D_{\mathrm{L}} / D_{\mathrm{S}}<0.103 ; 0$ $0.083 \leqslant D_{\mathrm{L}} / D_{\mathrm{S}}<0.093 ;-0.103 \leqslant D_{\mathrm{L}} / D_{\mathrm{S}}<0.113$. The width of these intervals reflects the experimental error in $D_{L} / D_{S}\left(\Phi, c_{S}\right)$ of $5 \% \quad 134]$ 
free parameter by an analytical formula using the MDA [25] or the renormalization scheme proposed by Alexander et al. [15].

We now turn to the diffusional properties of colloidal solids. For the crystalline phase two morphological modifications were investigated [33] and the results arc also shown in Fig. 2. The self diffusion in the monolithic crystals is below the resolution of our FRS set-up ( $D_{\mathrm{L}} \leqslant$ $4 \cdot 10^{8} \mathrm{~m}^{2} \mathrm{~s}^{-1}$ ) and we only give an upper limit. In the polycrystalline modification, however, the diffusion ratio is not more than two orders of magnitude below the value of the melt. In striking contrast to the former fluid case, it increases by roughly an order of magnitude with increasing strength of interaction.

The feature coincides with a significant decrease in crystallite size, which changed from $3 \mathrm{~mm}$ at the phase boundary to $20-30 \mu \mathrm{m}$ at $\Phi=0.0041$ and completely deionized conditions. For the samples of strongest interaction the volume of the grain boundaries therefore may well reach some $10 \%$. Accordingly, the observations can successfully be explained by grain boundary diffu sion, which increases with increasing grain boundary volume [33].

The observed small difference in the diffusion ratios between solid and molten phase underlines the nanocrystalline nature of the sample. This trend was also observed in hard-sphere suspensions (PMMA particles in decaline at $\Phi \geqslant 0.536)[2,36]$, but was interrupted at the volume fraction of $\Phi \approx 0.63$, roughly corresponding to that for the random dense packing of hard spheres. Suspensions of higher $\Phi$ show glass-like structure and dynamics. In our case, we do not expect a glass transition to occur since the volume fraction is still extremely low [31]. Preliminary experiments indicate instead that further increase in $\Phi$ may first lead to a highly diffusive solid state showing an amorphous-like structure factor, before the glassy state is reached.

\section{Morphology dependent elastic properties}

The study of the elastic and plastic response of colloidal solids subjected to external shearing fields $[37-43,11$, $16]$ is motivated by the long-term perspectives to gain access to very general concepts for the deformation and processing of crystalline material on a microscopic scale [44]. In this chapter, we will try to demonstrate that the known basic concepts may well be applicable quanlitatively, although colloidal solids have a number of unique features as compared to atomic matter. Furthermore, we will show that the specific advantages of colloidal solids may provide a rich source of enhanced understanding of important details neither directly adjustable nor even observable in other systems.
The elastic behavior of colloidal solids is determined by features already observed in disordered samples and retained over the phase transition [11]. Due to the low particle densities of $\rho \approx 10^{17}-10^{19} \mathrm{~m}^{-3}$ the bulk and shear moduli are in the range of only $0.1-10 \mathrm{~Pa}$ as compared to GPa for atomic solids. Therefore samples placed in resonator cells of $\mathrm{cm}$ size can be excited to resonant shear vibrations in the $1-100 \mathrm{~Hz}$ range. Due to the presence of the viscous suspending medium, however, higher harmonics and longitudinal modes are strongly overdamped and do not propagate.

We now turn to the influence of morphological details [45]. The intrinsically anisotropic elastic constants are not averaged out for textured polycrystalline material and the numerical averaging coefficients for unoriented samples depend on the size distribution as well as on the crystallite form.

These effects, however, are much less pronounced than the influence of variations in the interaction or the sample structure. We therefore developed a detection scheme which applies time-resolved static light scattering to measure the shear modulus $G$ with sufficient accuracy [45]. The resonance spectra of samples mechanically excited to torsional vibrations are monitored by recording the shift of the Bragg-condition observed under the oscillatory deformation of the crystal lattice. The new technique meanwhile has also been applied to systems sheared by electric or flow fields [46, 47]. Knowing the sample geometry, the shear modulus $G$ is derived from the resonance frequencies. In turn, $G$ is connected to the pair energy of interaction $V(r)$ in the harmonic approximation $[44,11,16]$ :

$$
G=f_{\mathrm{a}} f_{\mathrm{s}} \rho \frac{d^{2} V(r)}{d r^{2}}=f_{\mathrm{a}} f_{\mathrm{s}} \rho \varkappa^{2} r^{2} V(r)
$$

Here, $r$ denotes the mean interparticle distance and $f_{\mathrm{s}}$ and $f_{\mathrm{a}}$ are known numerical factors [16] depending on the structure of the crystallites and the average of their relative orientation as compared to the direction of applied shear. Under the assumption of randomly oriented polycrystalline material this relation has formerly been used to determine Yukawa parameters from fits to experimental data [11]. For our samples the Yukawa parameters are known and precisely adjustable. Also, the morphology can be carefully controlled by static light scaltering and microscopy. Since the method detects a $2 \%$ variation of the shear modulus, even when $G$ is in the range of $G \sim 0.1 \mathrm{~Pa}$, it can here be used to test $\mathrm{Eq}$. (3) and, furthermore, to discriminate even slightest morphology-induced effects.

In Fig. 4, we compare theoretical predictions based on the MDA and Eq. (3) for the salt concentration dependence of $G$ to experimental data on samples of selected morphologies [45]. The lines represent the values ex- 


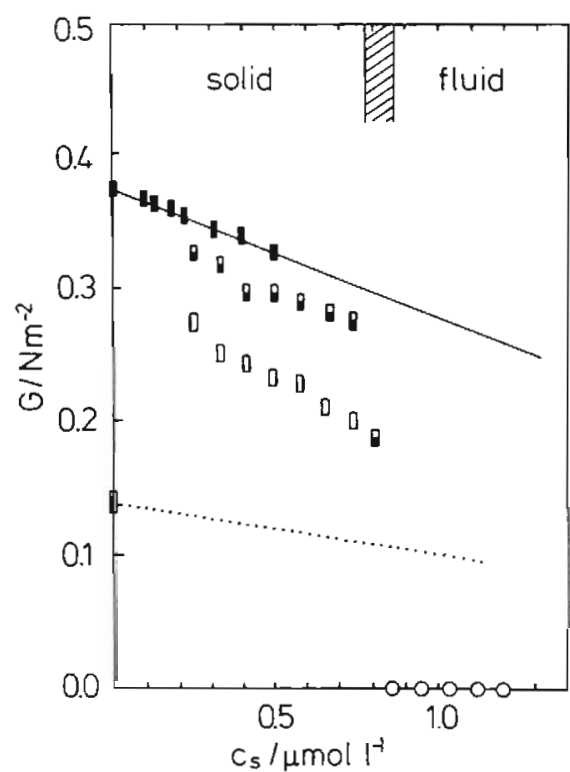

Fig. 4 Salt concentration dependence of the shear modulus $G$ of a suspension of $\Phi=0.0028$. The symbols refer to the experimental data for samples of different morphology. Filled rectangles: randomly oriented polycrystalline; half-filled rectangles: monolithic wall crystal - polycrystalline core; open rectangles: orjented polycrystalline; vertically striped rectangle: monolithic crystal oriented favorably for shearing; open circles: fluid samples. The lines refer to theoretical calculations: (-) fit of Eq. (3) with $f_{\mathrm{A}}=$ 0.5 (randomly oriented polycrystals) to the data at low salt concentration using a constant renormalized charge number of $Z^{*}=395$ as only fit parameter. ( $\cdots$ ) prediction of $G$ using the same parameters, but for a single crystal oriented with | III| parallel to the applied shear. A]so included are the observed freezing and melting lines indicating the coexistence region (hatched) [45]

pected for unoriented polycrystalline material and for a single crystal oriented in the $\langle 111\rangle$ direction parallel to the applied shear. In general, the shear moduli decrease roughly by some $30 \%$ from the completely deionized state to the phase boundary, where $G$ drops to zero very quickly. The last point of finite $G$ is measured in the coexistence region. The fluid phase does not support low frequency shear resonances.

We observe an excellent agreement between the values of $G$ for these two morphologies and the theoretical prediction which contains no free parameter. The description fails for the point measured at the coexistence of percolated randomly oriented crystallites immersed in a surrounding fluid phase. The very poor reproducibility of $G$ in successive melting-freezing cycles at this point indicates the statistical nature of the percolation details. For the wall crystal/polycrystalline core morphology a significant constant shift towards lower shear moduli is observed, while the overall dependence on the salt concentration is retained. This is not the case if the samples are subsequently subjected to shear of moderate amplitude which causes shear-induced alignment. With increasing salt concentration the samples show increasingly pronounced texturing after equal shear treatment. Their shear modulus steadily approaches the values for the favorably oriented single crystal.

Equation (3) presented a particularly simple concept for representative morphologies, which was taken from the physics of condensed atomic matter. Although colloidal solids posses additional unique properties, the experiments clearly show the the applicability of these models for some selected sample morphologies. Nevertheless, systematic deviations are clearly visible for other modifications and additional corrections are necessary to represent their influence. With colloidal solids, such modifications in Eq. (3) can easily be developed and tested, because a wide variety of modifications may be prepared by controlled solidification and subsequent shear-processing. Those can be carefully characterized with respect to grain size and form distributions, orientation correlation lengths, character of grain boundaries and inclusions, among others.

\section{Resonant stick-slip motion}

The experiments presented in the preceding section were performed under conditions of very low shear amplitudes. The maximum displacement of particles from their equilibrium position was restricted to less than $1 \%$ of the nearest neighbor spacing in order to retain the crystal morphology. At larger elongations the solid will creep and show plastic flow [46] which has already been exploited in the preparation of different morphologies. At large Deborah number $D e=\dot{\gamma} r^{2} / 4 \pi D_{\mathrm{f}} \approx 0.2$, which gives the ratio of shear rates $\dot{\gamma}$ to (diffusive) structural relaxation times [48], a non-equilibrium melting transition is observed. Note that the value of De corresponds to the value of the Lindemann criterion, predicting a melting transition if the thermal elongation exceeds $0.1-0.2 \mathrm{r}$. The transition is predicted to be reentrant at high interaction [48]. Experimentally, a succession of different phases is observed before the shear-molten state is reached, which depends critically on the shear geometry. The scenario includes, for example, not only layered hexagonal sheets or strings [49], but also more complicated structures $[4,11]$.

The example of shear-induced melting presented in this section is simple in terms of the non-equilibrium phase diagram, since it shows a direct transition from the solid to the fluid state without a detectable intermediate phase. It nevertheless is of great interest, since here for the first time a resonant coupling of a slip-stick motion to an internal vibration of one solid sliding over another is observed on a microscopic level [47].

A monolithic crystal oriented favorably for shearing is grown in an open-ended rectangular resonator cell of 
Fig. 5 Polarization microscopic picture of a suspension at $\Phi=0.003$ and $c_{\mathrm{s}}=0$ solidified in the presence of a shearing field. The bimodal size distributions shown were prepared by varying the amplitude and frequency of the field

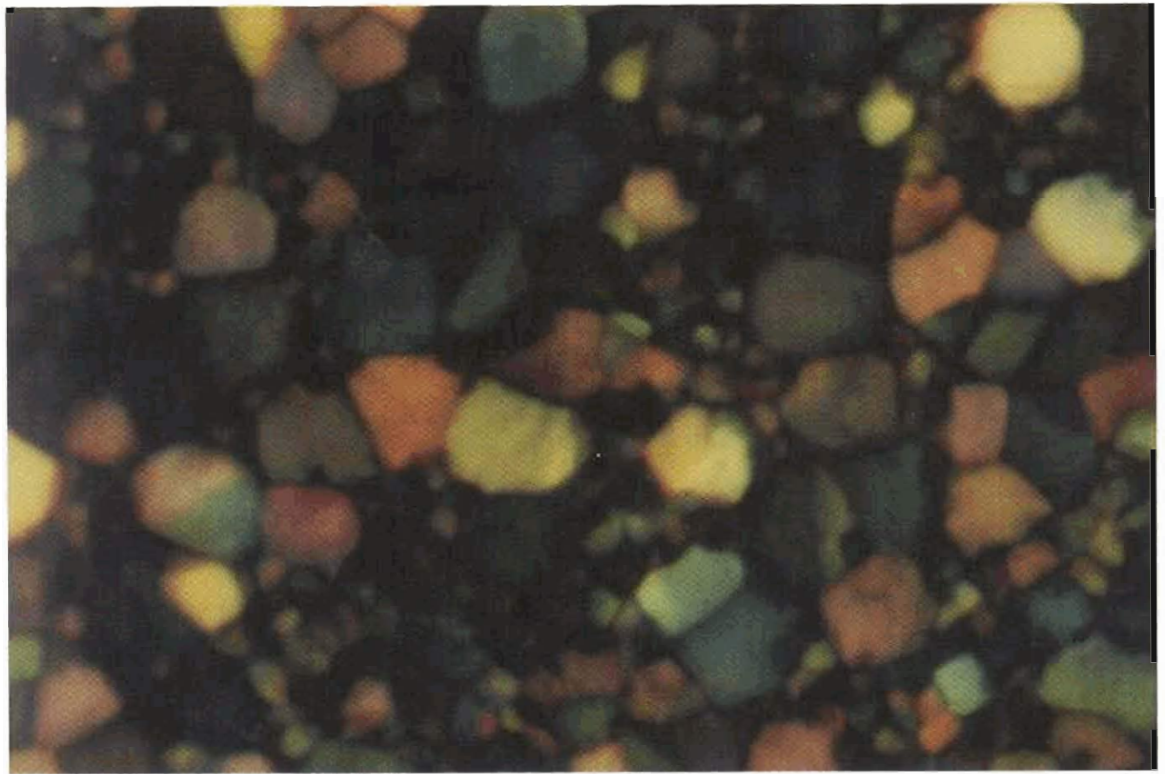

dimensions $1 \times 10 \mathrm{~mm}$. Using time-resolved static light scattering the resonance frequency of thermal-noise-induced vibrations was determined to be $14 \mathrm{~Hz}$ corresponding to a shear modulus of $0.14 \mathrm{~Pa}$. If a hydrostatic pressure difference is applied the crystal is pushed through the cell. However, resonances are also observed under flow. A mode at $7 \mathrm{~Hz}$ corresponds to the vibrating crystal which now is fixed only at one wall, while it sways foreward at the opposite side of the cell. At the point of largest elongation the crystal is pinned again and the process is repeated with exchanged fixed and loose sides. The vibrational mode is superimposed on an asymmetrically broadened peak at $3.5 \mathrm{~Hz}$, which represents this alternating periodicity of the slip-stick motion. The crystal moves without melting in a "swingingduck-walk" fashion.

At a critical velocity the crystal depins from both walls and starts gliding, lubricated by thin layers of fluid phase. Both resonances are no longer excited. With increasing velocity the fluid layers widen and at a second critical velocity complete shear melting is monitored by the disappearance of the Bragg reflections.

Slip-stick motion is of interest in a wide field of mechanical applications and constitutes an important mechanism of friction. It was recently observed on molecular scale between surfaces separated by a molecularly thin lubrication layer [50]. The results reflected the discrete, molecular nature of the intermediate fluid. No resonant coupling to vibrational modes was observed, as might be expected considering the large difference in time scales of the slip-stick periodicity and of acoustic resonances of solids. Coincident time scales are present in the macroscopic process of bowing a string instrument, where the slip-stick motion of the bow is synchronized to the resonant vibration of the string. They are also present in the colloidal crystal (violin) which provides the first suitable model system for such phenomena on a microscopic scale.

\section{Quasi-epitaxial growth of colloidal crystals}

The preceding sections have demonstrated the importance of controlling the sample morphology. As an alternative technique to shear-processing, controlled solidification may be used to adjust, for example, the grain size or the orientation. Under conditions of homogeneous nucleation the crystallite size depends on the strength of interaction, which increases with increasing volume fraction and charge, and also with decreasing screening [51]. The higher the nucleation rate the earlier the crystal growth is terminated, i.e., large undercooling produces small crystals. Additional modifications may be introduced if the nucleation process is performed under shear. The details of the resulting decrease of nucleation rate densities are not fully understood, but as an example, we show a sample with bimodal grain size distribution in Fig. 5. It is a promising candidate to study the influence of inclusions and their percolation on the shear modulus.

On the other hand, homogeneous nucleation can be suppressed kinetically by the simultaneous growth of heterogeneous nucleated crystals on a suitable substrate. Even in the shear-molten state next to the cell walls a finite layer of hexagonal planes [52] is observed, which are oriented with their $\langle 111\rangle$ directions parallel to each other and to the flow direction. After termination of shear flow this structure serves as a nucleus for the now quasi- 


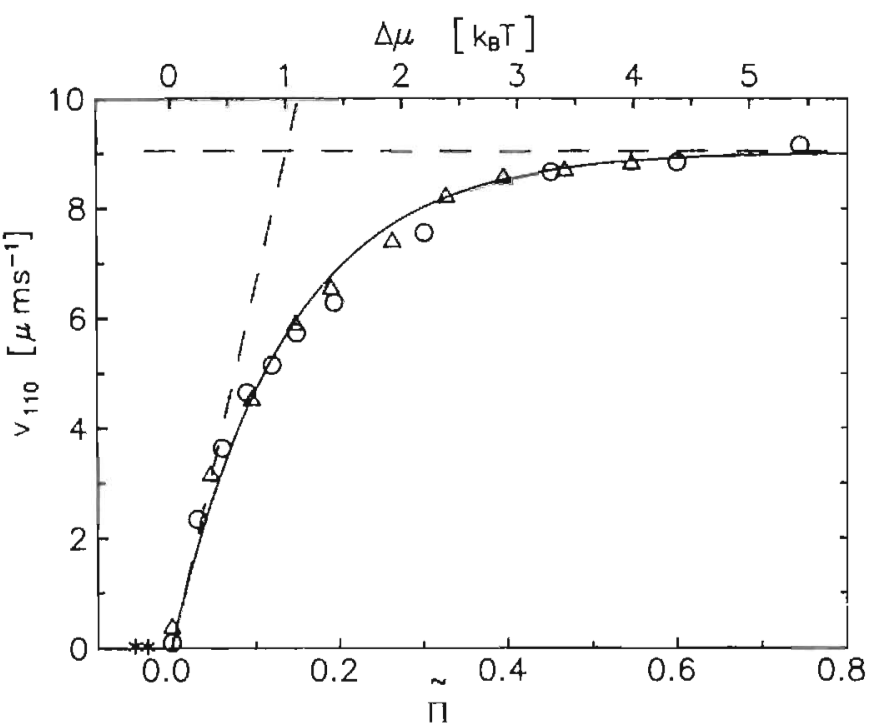

Fig. 6 Growth velocity $v_{110}$ of a bcc monolithic crystal in the (110) direction in dependence on the parameter $\tilde{\Pi}$. The upper scale shows the corresponding chemical potential difference between melt and solid $\Delta \mu=\mathrm{B} \Pi$ as derived from the fit. The symbols refer to measurements in dependence on the volume fraction at completely deionized conditions (squares) and on the salt concentration at $\Phi$ $=0.0030$ (circles). The solid line is a fit of Eq. (5) to the experimental data. The dashed lines are guides for the eye indicating the value of the limiting velocity $v_{\infty}=9.05 \mu \mathrm{m} \mathrm{s}^{-1}$ and the initially linear increase $\left(B=7.4 k_{\mathrm{B}} T^{\prime}\right)[29]$

epitaxially growing monolithic crystal which retains the orientation of the starting layers. The phenomenon already has been used to decorate flow patterns of TaylorCouette instabilities in undercooled colloidal melts $[11 \mathrm{j}$. The term monolithic is used to point out that the oriented crystal contains cloudlike lateral twin patterns caused by strain relaxation processes following the transformation of hexagonal layers into the [110] planes of the bcc lattice. Careful analysis of Bragg reflections in both $k$ - and $r$-space reveal a vertical stacking of pancake like twin domains [53].

While the recent experiments concerning the shear processing of colloidal solids aim at extensive studies of the non-equilibrium phase diagram and the application to controlled preparation of particular morphologies, experiments on the controlled solidification after shear already proceed a step further towards a quantitative understanding. Earlier experiments qualitatively showed that the growth velocities of colloidal crystal increase with increasing interaction $[5,21]$ and for some systems at large volume fractions (e.g., in hard sphere suspensions) the dominant interplay between entropy and mass diffusion was quantitatively demonstrated $[1,21,54,55]$. An interesting question, however, remains, namely, what exactly drives and limits the growth in charge stabilized dilute systems.
The growth velocities were measured by time-resolved static light scattering under an angle off the Bragg condition providing large differences in scattered intensity for the respective phases [26]. A laser bcam crossing the cell containing the quasi epitaxially growing crystal was observed with a long distance telescopic microscope giving direct information about the evolution of the crystal length. Linear growth was found for all conditions within the experimental error of $2 \%$.

We performed several runs over a large part of the bcc region of the phase diagram. In particular, the dependence on the salt concentration and the particle density were determined independently. Since we know the coordinates of the equilibrium phase boundaries [29] and of the samples under study [28], and further possess an independently tested description of the pair interaction $[26,30]$, we can now try to evaluate measured growth velocities in terms of well-defined quantities. The derived growth velocities $v_{10}$ are plotted in Fig. 6 over the quantity:

$\tilde{\Pi}=\frac{\Pi-\Pi_{\mathrm{m}}}{I I_{\mathrm{m}}}=\frac{\left(\rho V(r)-\rho_{\mathrm{m}} V(r)_{\mathrm{m}}\right)}{\rho_{\mathrm{m}} V(r)_{\mathrm{m}}}$,

where the index $m$ refers to the melting line [26]. The measured data collapse onto a single curve, indicating that $\tilde{\Pi}$ nicely captures both the volume fraction dependence and the influence of enhanced screening. The plot shows an initially linear increase of $v_{110}$ with in. creasing $\tilde{\Pi}$ which saturates at a plateau valuc. For a wide range of experimental parameters the data are excellently described by a Wilson Frenkel growth law:

$v_{100}=v_{\infty}\left[1-\exp \left(\Delta \mu / k_{\mathrm{B}} T\right)\right]$

with $\Delta \mu=B \tilde{\Pi}$. The best fits for $B$ give $B=7.4 \pm 0.1$ $k_{\mathrm{B}} T$. The only exeption is found for the points measured in coexistence corresponding to negative $\tilde{\Pi}$. The quality of these fits to the high precision data indicates that our formulation of $\tilde{\Pi}$ quantitatively captures the relevant phenomena. We conclude that in contrast to hard sphere systems at high volume fraction, entropic effects play only a negligible role. The transition is driven by the difference in the potential energy density, which may be interpreted as a reduced osmotic pressure difference.

For large overpressure the velocity approaches a limiting value of $v_{\infty}=9.05 \pm 0.1 \mu \mathrm{m} \mathrm{s}^{-1}$. This value is diffusion controlled in the sense that at large overpressures the surface of the growing crystal is an extended layered structure formed by diffusional processes. The structure closely resembles layered structures observed adjacent to the cell wall and the estimated lower limit of its thickness is three hexagonal layers [29]. The proposed mechanism is fundamentally different from mass diffusion control [55] at clevated volume fractions, since the difference in density between crystal and melt here is below experimental resolution $\left(v_{110}(t)=\right.$ const. $)$. 
Under conditions isolating the growth process from the nucleation problem we therefore could demonstrate the validity of comparably simple concepts from atomic solid state physics. Our current interest is in an equally quantitative understanding of nucleation rate densities in order to form a basis for studies on the slowed nucleation and growth dynamics as well as on changes in the phase diagram observed under shear, and which seem to be strongy connected to phenomena occurring in ball milling or geological processes.

\section{Conclusions}

Colloidal solids formed from dilute monodisperse latex spheres strongly interacting via a screened Coulomb potential only form a small and very specialized system within the large and heterogeneous field of colloidal dispersions. We have demonstrated that, nevertheless, results derived from these model suspensions allow for generalizing not only for other colloidal systems, but also towards general problems of condensed matter physics.

The prime advantages of these solids are: i) the precise control of the pair interaction and its consistent description, facilitating quantitative tests of microscopic models for elasticity, crystallization kinetics or self-diffusion; ii) the unique possibility of simultaneous high temporal and spatial resolution via non-destructive optical techniques, and iii) the possibility of preparing representative morphologies by controlled solidification and shear processing.

A number of open questions like the modeling of shear processing, the non-equilibrium phase behavior or the influence of morphological details on dynamical quantities can now be adressed on the basis of a well understood equilibrium system and a careful discrimination of colloid specific properties.

Acknowledgements We are indebted to H. Löwen, L. Belloni, R. Klein, K. Schätzel and R. Piazza for many inspiring discussions and to $R$. Beck for her help with the artwork. We gratefully acknowledge the financial support of the Deutsche Forschungsgemeinschaft (SFB 306 TP C4 \& Pa459-1).

\section{References}

1. Chen SH, Huang JS, Tartaglia P (eds) (1992) Structure and Dynamics of Strongly Interacting Colloids and Supramolecular Aggregates in Solution. Kluwer Academic Publishers, Amsterdam, VATO ASI Series Vol C369

2. Pusey PN (1991) In: Hansen JP, Levesque $\mathrm{D}$, Zinn-Justin $\mathrm{J}$ (eds) Liquids, freezing and the glass transition, Ecole d'ete, Les Houches, 5lst, Elsevier, Amsterdam, p 763

3. Safran SA, Clark NA (eds) (1987) Physics of Complex and Supramolecular Fluids, Wiley-Interscience, New York

4. Hirtzel CS, Rajagopalan R (1985) Colloidal Phenomena: Advanced Topics, Noyes Publishers, Park Ridge, NJ and Salgi P, Guerin J-F, Rajagopalan R (1992) Colloid Polym Sci 270:785

5. Aastuen DJW, Clark NA, Cotter LK, Ackerson BJ (1986) Phys Rev Lett 57:1733

6. Gast AP, Monovoukas Y (1991) Nature 351:553

7.Dozier WD, Chaikin PM (1982) J Physique 43:843

8. Ackerson BJ, Chowdbury AH (1987) J Chem Soc Faraday Discuss 83:1

9. Xue WG, Grest GS (1990) Phys Rev Lett 64:419

10. Tata BVR, Rajalakshmi M, Arora AK (1992) Phys Rev Lett 69:3778

11. Chaikin PM, DiMeglio JM, Dozier WD, Lindsay HM, Weitz DA, in [3], p 65
12. Cotter LK, Clark NA (1987) J Chem Phys 86:66l6

13. Schätzel K, Ackerson BJ (1992) Phys Rev Lett 68:337

14. Murray CA, Van Winkle DH (1987) Phys Rev Lett 58:1200

15. Alexander $S$, Chaikin PM, Grant $P$, Morales GJ, Pincus P, Hone D (1984) J Chem Phys 80:5776

16. Robbins MO, Kremer K, Grest GS (1988) J Chem Phys 88:3286

17. Voegtli LP, Zukoski ChFIV (1991) J Coll Interface Sci 141:79

18. Oxtoby DW, Evans RJ (1988) J Chem Phys 89:7521

19. Löwen H, Hansen JP, Roux JN (1991) Phys Rev A 44:1169

20. Klein R, in [1], pp 39

21. Ackerson BJ (ed) (1990) Phase Transitions $21: 2-4$

22. Bucci S, Fagoti C, Degiorgio V, Piazza R (1991) Langmuir 7:824

23. van Blaaderen A, Peetermanns J, Maret J: Dhont JKG (1992) J Chem Phys 96:4591

24. Palberg T, Mönch W, Piazza R, Bellini $T$, Birzer F (1994) Phys Rev Lett, submitted

25. Dozier WD, Lindsay HM, Chaikin PM (1985) J Physique Collog 46-C3:257

26. Bitzer F, Palberg $T$, Löwen $H$, Simon R, Leiderer P (1994) Phys Rev E, accepted

27. Palberg T, Würth $M$ (1994) Phys Rev Lett $72: 786$

28. Palberg $T$, Härtl $W$, Wittig $U$,
Versmold $H$, Würth $M$, Simnacher $E$ (1992) J Phys Chem 96:8180

29. Würth M, Palberg T, Schwarz J, Culis F, Leiderer P (1994) J Chem Phys, submitted

30. Palberg T, Kottal J, Bitzer F, Simon R, Würth M, Leiderer P (1994) J Coll Interface Sci (in press)

31. Sirota EB, Ou-Yang HD, Sinha SK, Chaikin PM, Axe JD, Fujii Y (1989) Phys Re Lett 62:1524

32. Meier EJ, Frenkel D (1991) J Chem Phys 94:2296

33. Simon R, Palberg T, Leiderer P (1993) J Chem Phys 99:3030

34. Löwen H, Palberg T, Simon R (1993) Phys Rev Lett 70:1557

35. Löwen H (1994) Physics Reports 334: 1

36. Van Megen W, Pusey PN, Bartlett $P$, in [21], pp 207

37. Crandall RS, Williams S (1977) Science 198:293

38. Dubois-Violette E, Pieranski P, Rothen F, Strzlecki L (1980) J Physique 41:369

39. Lindsay HM, Chaikin PM (1983) J Chem Phys 76:3774

40. Lindsay HM, Chaikin PM, In: [4], pp 269

41. Joanicot $M$, Jorand $M$, Pieranski $P$, Rothen F (1984) J Physique 45:1413

42. Joanny JF (1979) J Coll Interface Sci $71: 622$

43. Jorand M, Dubois-Violette E, Pansu B, Rothen F (1988) J Physique 49: 1119

44. Cahn RW, Haasen R (1983) Physical Metallurgy, Amsterdam

45. Palberg T, Kottal J, Loga T, Hecht H, 
Simnacher E, Falcoz F, Leiderer $P$ (1994) J Physique III 4:31

46. Würth M, Palberg T (1994) J Coll Interface Sci, 167 (in press)

47. Palberg T, Streicher K (1994) Nature 367:51

48. Stevens MJ, Robbins MO, Belak JF (1993) Phy's Rev Lett 66:3004
49. Ackerson $\mathrm{BJ}$ in |31, pp 553

50. Gee ML, McGuiggan PM, Israelachvili JN, Jomola AM (1990) J Chem Phys 93: 1895

51.Palberg T, Schwarz J, Mönch W. Leiderer PJ (to be published)

52. Grier DJ, Murray CA in [I], pp 145
53. Palberg $T$, Würth $M$, König $P$, Schwarz $J$, Leiderer $P$ (to bc published)

54. Poon WCK, Selfe JS, Robertson MB, Illett SM, Pirie AD, Pusey PN (1993) J Physique II 3:1075

55. Gast AP, Monovoukas Y (1991) Nature $351: 553$ 